\title{
Recomendações sobre exposição aos riscos ocupacionais pela equipe de enfermagem: uma revisão integrativa
}

\author{
Recommendations for exposure to occupational risks by \\ nursing team: an integrative review
}

Renata Vieira Girão Arcanjo' • Barbara Pompeu Christovam² • André Luiz de Souza Braga³

\section{RESUMO}

Objetiva-se mapear o perfil bibliométrico das produções sobre riscos ocupacionais na equipe de enfermagem, e identificar as recomendações apontadas pelos artigo,s para redução ou eliminação da exposição aos riscos ocupacionais pela equipe de enfermagem. Trata-se de uma revisão integrativa a partir de publicações científicas de enfermagem indexadas nas bases de dados: LILACS, MEDLINE, BDENF e IBECS. Foram selecionados para análise 28 estudos, dos quais 21 apresentavam como cenário unidades hospitalares; enquanto 5 estudos foram realizados na Atenção Básica a Saúde. Os riscos biológicos lideraram os temas dos estudos com 14 ocorrências (50\%).A principal recomendação apontada pelos estudos foi a realização de intervenções educativas. Concluí-se que a partir deste estudo, as pesquisas desenvolvidas posteriormente poderão ser inspiradas a investirem seus esforços em testarem as recomendações aqui apontadas, para que haja maior compreensão de sua eficácia.

Palavras-chave: Enfermagem; Gestão da Segurança; Riscos Ocupacionais.

\begin{abstract}
The aim is to reach the bibliometric indicators of production on occupational hazard to the nursing staff; and to identify the recommendations in scientific articles for the lessening or elimination of the occupational hazard the nursing staff is exposed to. An integrative review based on nursing scientific publications using LILACS, MEDLINE, BDENF and IBECS database. Have been selected for analysis 28 studies, from which 21 were hospital units; whereas 5 of them were undergone in basic care. Bio-risk is the main topic of the studies due to the high level of occurrence, 14 incidents (50\%). The main recommendation from the studies was educational intervention. It concludes that from the present study, previously developed researches will be able to find the incentive to put their recommendations to the test so their effectiveness is widely understood.
\end{abstract}

Keywords: Nursing; Safety Management; Occupational Risks.

${ }^{1}$ Mestre em Ciências do Cuidado em Saúde pela Escola de Enfermagem Aurora de Afonso Costa da Universidade Federal Fluminense-UFF. Enfermeira formada pela UFF. Atuou na Emergência do Hospital Otime Cardoso dos Santos em Cabo Frio. Atualmente encontra-se em Dublin, Irlanda, realizando intercâmbio para aprimoramento da língua inglesa.E-mail: renatavg@id.uff.br

2 Doutora pelo Programa de Pós-Graduação da Escola de Enfermagem Anna Nery (2009). Docente permanente do Programa de Mestrado Profissional em Enfermagem Assistencial e do Programa de Mestrado Acadêmico em Ciências da Saúde da EEAAC/UFF, Diretora do Centro de Atenção e Investigação em Tuberculose e Doenças Pulmonares Profo Mazzine Bueno da UFF, Coordena o Curso de Especialização em Gerência dos Serviços de Enfermagem da EEAAC/UFF, Vice-Líder do Grupo de Pesquisa Cidadania e Gerência na Enfermagem. Terceira Coordenadora Geral da Rede Internacional de Gestão do Cuidado da OPS/OMS. E-mail: babypompeu@gmail.com

${ }^{3}$ Doutorando do Programa de Doutorado em Ciências do Cuidado em Saúde da Escola de Enfermagem Aurora de Afonso Costa. Mestre em Ensino de Ciência da Saúde e do Ambiente pelo UNIPLI (2005). Atualmente professor do $3^{\circ}$ Grau da Universidade Federal Fluminense - UFF. Tem experiência na área de Saúde Coletiva, com ênfase em Epidemiologia, atuando principalmente nos seguintes temas: gerência em enfermagem, epidemiologia, gestão em saúde, educação saúde, informação em saúde e odontologia.E-mail: andre.braga@globo.com 


\section{INTRODUÇÃO}

A Organização Mundial de Saúde (OMS) relata que cerca de $45 \%$ da população mundial e cerca de $58 \%$ da população acima de 10 anos de idade faz parte da força de trabalho. 0 trabalho desta população sustenta a base econômica e material das sociedades, que por outro lado são dependentes da sua capacidade de trabalho. Esses trabalhadores garantem 0 desenvolvimento socioeconômico e sustentável de seus países ${ }^{1}$.

0 trabalho é crucial para a subsistência humana. E assim sendo, merece ser desenvolvido em condições dignas, e em ambientes saudáveis, garantindo a produtividade necessária. Entretanto, os indicadores de acidentes de trabalho revelam as condições indignas e insalubres a que estão sendo submetidos tais trabalhadores.

No Brasil, os dados da Previdência Social apresentados em seu anuário estatístico revelam que no ano de 2013 o setor de atividade econômica "Saúde e Serviços Sociais" apresentou um total de 70.602 casos de acidentes de trabalho e a segunda maior participação nos acidentes típicos com $12,08 \%$ do total².

Dentre o grupo de profissionais que compõe a equipe de saúde encontra-se a equipe de enfermagem, que agrega diferentes categorias profissionais e que se expõe a riscos ocupacionais rotineiramente. Esta exposição relacionase ao contato direto dos trabalhadores de enfermagem durante a assistência aos pacientes, além do tipo e da frequência de procedimentos realizados.

Os riscos ocupacionais presentes ou relacionados ao trabalho dos profissionais de enfermagem são classificados, de acordo com a Organização Pan-Americana da Saúde no Brasil (OPAS), em cinco grandes grupos: físicos; biológicos; químicos; ergonômicos; e psicossociais e de acidentes ${ }^{3}$.

Os riscos ocupacionais afetam a segurança, a saúde humana, a integridade profissional e a imagem institucional, e podem ocasionar acidentes de trabalho, doenças ocupacionais e até mesmo a morte, sendo imprescindível que sejam adotadas medidas de redução e controle de tais riscos no ambiente do trabalho destes profissionais.

Assim, na busca por recomendações eficazes para os profissionais de enfermagem sobre os riscos ocupacionais, foi realizada a presente revisão integrativa, com o objetivo de mapear o perfil bibliométrico das produções sobre riscos ocupacionais na equipe de enfermagem, e identificar as recomendações apontadas pelos artigos para redução ou controle da exposição aos riscos ocupacionais pela equipe de enfermagem.

\section{MÉTODO}

Trata-se de um estudo de natureza qualitativa no seguimento das revisões bibliográficas sistemáticas, utilizando como método a revisão integrativa ${ }^{4}$. Adotouse a revisão integrativa da literatura por seu perfil sistematizador na busca de produções e na análise dos resultados, visando à compreensão do tema proposto, a partir dos estudos realizados.

Para a elaboração da presente revisão integrativa, as seguintes etapas foram percorridas: estabelecimento do objetivo da mesma; definição das bases de dados a serem utilizadas; estabelecimento de critérios de inclusão e exclusão de artigos; definição das informações a serem extraídas dos artigos selecionados; análise dos resultados; discussão e apresentação dos resultados e elaboração da conclusão ${ }^{4}$.

Para guiar a revisão integrativa, formulou-se a seguinte questão: quais as recomendações apontadas nas produções científicas voltadas para diminuição ou controle da exposição aos riscos ocupacionais pela equipe de enfermagem?

Para a seleção dos estudos foram utilizadas as seguintes bases de dados: Literatura Latino-Americana e do Caribe em Ciências da Saúde (LILACS); Literatura Internacional em Ciências da Saúde (MEDLINE); Base de Dados de Enfermagem (BDENF); Índice Bibliográfico Espanhol de Ciências da Saúde (IBECS). Os descritores em ciências da saúde (DeCS) utilizados foram: Nursing, Safety Management e Occupational Risks. A busca foi realizada entre os dias 22 de outubro de 2014 e 05 de novembro de 2014.

Os critérios de inclusão foram: produções que abordassem os riscos ocupacionais nos profissionais de enfermagem, produções que abordassem os riscos ocupacionais individualmente ou em conjunto, produções que sugerissem estratégias para diminuição ou controle dos riscos ocupacionais. Os filtros utilizados foram: produções com texto completo e disponíveis online; publicados nos últimos cinco anos; disponíveis nos idiomas inglês, espanhol e português. Os critérios de exclusão foram: publicações que não descrevessem a metodologia utilizada.

Para a análise e posterior síntese dos artigos selecionados foi utilizada uma matriz de síntese especialmente construída para esse fim. As informações extraídas dos artigos foram: idioma, abordagem, método utilizado, cenário do estudo, tipo de risco abordado, e as recomendações para a diminuição ou controle da exposição aos riscos ocupacionais pela equipe de enfermagem, que foram relacionadas, e posteriormente categorizadas. Segue abaixo o fluxograma das buscas.

\section{RESULTADOS}

Após percorrer todas as etapas metodológicas estabelecidas na revisão integrativa da literatura, foram selecionadas vinte e oito produções científicas. Das quais vinte e quatro $(85,7 \%)$ estavam disponíveis na língua portuguesa, três $(10,7 \%)$ disponíveis em língua inglesa e uma $(3,5 \%)$ disponível em língua espanhola.

A abordagem quantitativa se fez presente em dezenove publicações $(67,8 \%)$, enquanto oito $(28,5 \%)$ apresentavam abordagem qualitativa, e apenas uma (3,5\%) a abordagem quanti-qualitativa. 


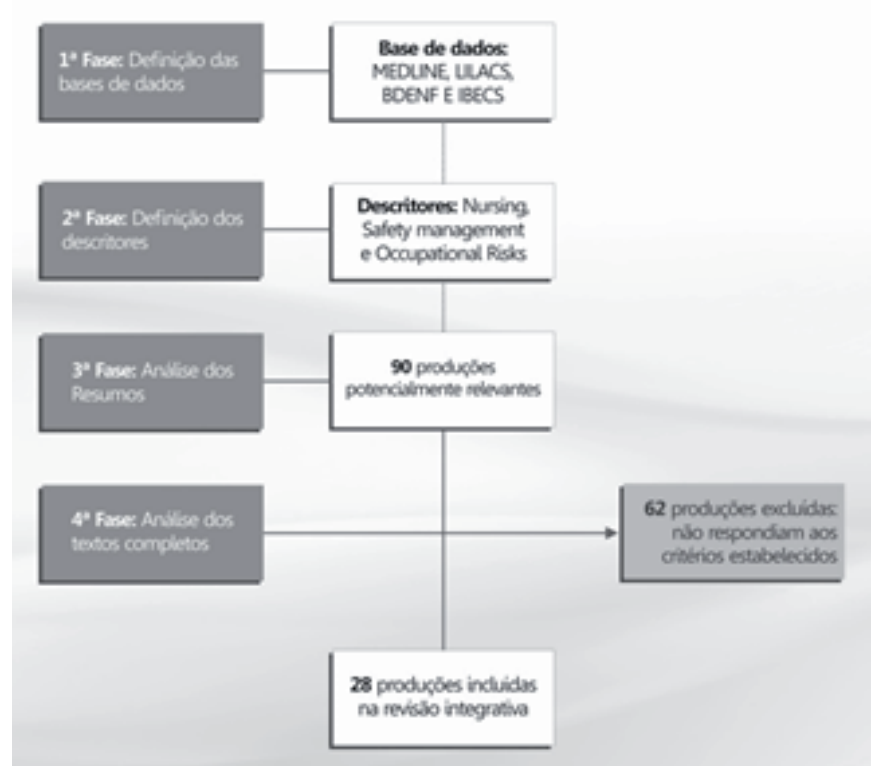

Fluxograma 1. Esquema representativo dos procedimentos de seleção dos estudos. Niterói, RJ, Brasil, 2014.

Fonte: dados da pesquisa.

Os tipos de método utilizados foram identificados separadamente. 0 método mais utilizado nas produções científicas foi o método descritivo, com 24 ocorrências (85,7\%),seguido do método exploratório com 10 ocorrências (35,7\%). Em seguida com 6 produções $(21,4 \%)$ aparece o método transversal, seguido do método epidemiológico com 4 publicações (14\%), e do método participante com 2 publicações (7\%). Os métodos: experimental; retrospectivo; estudo de intervenção; observacional; não experimental; correlacional e social; apareceram uma vez (3,5\%).

Houve discrepância no cenário de realização dos estudos. Vinte e uma publicações (75\%) realizaram seus estudos em unidades hospitalares, enquanto apenas cinco publicações (17,8\%) optaram pela Atenção Básica à Saúde, sendo que desses, dois (7\%) foram realizados em unidades de baixa complexidade e três $(10,7 \%)$ em unidades de média complexidade. Dois estudos $(7 \%)$ foram realizados em outros cenários de atuação da equipe de enfermagem.

Os tipos de riscos ocupacionais abordados na temática das publicações também foram contabilizados. Os riscos biológicos lideraram os temas dos estudos com 14 ocorrências (50\%). Em seguida, os riscos ocupacionais gerais, que traziam os riscos em conjunto, e não discutiam apenas um tipo de risco. Essa categoria contou com oito produções (28,5\%). Em seguida os riscos químicos com 4 ocorrências $(14,2 \%$ e es riscos ergonômicos e psicossociais sendo discutidos em 2 estudos (7,14\%).

Todos os estudos trouxeram recomendações para a redução ou eliminação da exposição aos riscos ocupacionais. As recomendações são de diversas naturezas e podem ser aplicadas nos diversos cenários de trabalho da enfermagem. Estas recomendações são consideradas pelos estudos como estratégias eficazes no combate às consequências da exposição aos riscos ocupacionais.
Dentre as recomendações sugeridas pelos estudos emergiram as seguintes categorias: Intervenções Educativas; Intervenções Estruturais; Equipamento de Proteção Individual; Participação dos trabalhadores; Realização de campanhas de Imunização; Melhoria nas condições do trabalho dos profissionais; e Estímulo a Notificação de Acidentes de Trabalho.

\section{DISCUSSÃO}

Os estudos produzidos em língua portuguesa foram mais expressivos quando comparados aos demais idiomas, e todos de origem brasileira. Este fato ocorre pela preocupação dos pesquisadores enfermeiros com a precária condição de trabalho vivenciada pela enfermagem no Brasil. A situação de trabalho, em alguns casos, sem as mínimas condições de segurança, perpassam os cenários das instituições públicas, alcançando também as instituições particulares, o que move a realização das pesquisas 5 .

As condições inadequadas de trabalho no país são descritas em diversos estudos. Este fator correlaciona-se a falta de investimento em melhores estruturas de proteção, além da falta de fiscalização das unidades de saúde e dos reflexos da condição econômica e social do país nas instituições de saúde ${ }^{5-6}$.

As pesquisas apresentam abordagem principalmente quantitativa, entretanto a estatística inferencial é utilizada na menor parte dos estudos. As pesquisas que trazem a abordagem quantitativa associada ao método descritivo são 16 produções, ou seja, $57 \%$ dos estudos analisados. Nota-se uma tendência entre essa associação, que pode ser justificada em função da vantagem que a pesquisa quantitativa descritiva tem na apresentação de seus achados.

Verifica-se a alta frequência de estudos realizados em cenário hospitalar (75\%), em detrimento de estudos em outros cenários de atuação. 0 ambiente de trabalho hospitalar é considerado uma grande área de risco de acidentes com alta frequência de realização de procedimentos, principalmente os que envolvem o contato direto com os pacientes ${ }^{5-7-8}$.

O hospital também é caracterizado pela realização de procedimentos invasivos e pelo contato dos profissionais com os pacientes em estado mais grave, além de possuir uma grande demanda de pacientes por serviços. Esses fatos tornam o ambiente hospitalar mais evidenciado para realização de estudos sobre riscos ocupacionais ${ }^{8-9}$.

O risco biológico é o risco ocupacional mais pesquisado em relação à equipe de enfermagem, englobando $75 \%$ das publicações desta revisão. Outra revisão integrativa que buscou publicações entre os anos de 2005 e 2010 também demonstrou que a principal preocupação nas pesquisas era o risco biológico ${ }^{10}$.

Estes dados revelam uma tendência para o desenvolvimento de pesquisas sobre riscos biológicos 
com a equipe de enfermagem. E pode ser correlacionada a escolha do cenário de desenvolvimento dos estudos, visto que o risco biológico será sempre encontrado em ambientes hospitalares, que também tiveram ampla frequência (75\%) nos estudos selecionados para esta revisão.

A constante exposição dos profissionais de enfermagem aos agentes biológicos é preocupante devido às peculiaridades dos procedimentos realizados no cuidado a saúde das pessoas, além de se destacarem quanto aos acidentes de trabalho ${ }^{11-3}$. A equipe de enfermagem desenvolve cerca de $60 \%$ das ações direcionadas aos clientes na prestação de assistência direta, o que torna o risco biológico mais evidente 9 .

Os riscos ocupacionais afetam a segurança dos profissionais e podem trazer graves consequências como a ocorrência de acidentes de trabalho e de doenças ocupacionais. Os acidentes são facilmente identificados, entretanto as doenças que surgem em decorrência do trabalho são silenciosas, e os agravos das mesmas podem ser tardiamente identificados, prejudicando a qualidade de vida do profissional em longo prazo. A fim de evitar tanto os acidentes como as doenças do trabalho, as recomendações dos estudos sobre riscos ocupacionais foram categorizadas e são apresentadas a seguir.

\section{Intervenções Educativas}

Nesta categoria foram listadas todas as recomendações que apontassem para alguma metodologia educativa. Vinte e quarto artigos $(85,7)$ apresentaram a intervenção educativa como uma proposta para a diminuição ou controle da exposição aos riscos ocupacionais. Os termos integrantes desta categoria variaram entre: educação permanente, educação continuada, ações educativas, capacitação, treinamento, dentre outros de similar conteúdo.

A educação continuada é fundamental para a qualidade de assistência de enfermagem, sendo como um processo de atualização técnico-científica contínua, promovendo o desenvolvimento pessoal e a autonomia no desempenho profissional ${ }^{14}$

A educação permanente em saúde também foi apresentada nos estudos como estratégia para redução da exposição aos riscos ocupacionais, e é definida como aprendizagem no trabalho, em que o aprender e o ensinar são incorporados ao ambiente profissional e propõe que a educação se faça a partir da problematização da própria prática $^{14}$.

Sabendo-se que os termos mencionados possuem diferenças metodológicas, cabe diferenciá-los e implementar no ambiente de trabalho a Educação Permanente focando os desafios da prática, assim como estimular a educação continuada dos profissionais para que se desenvolvam profissionalmente e possam contribuir com o local de trabalho através dos conhecimentos que tem adquirido.

\section{Intervenções Estruturais}

Nesta categoria estão inseridas as recomendações quanto a alterações na estrutura física dos setores. Nove publicações (32\%) mencionaram as intervenções estruturais como uma recomendação fundamental para a redução a exposição aos riscos ocupacionais. Algumas medidas como a reforma de bancadas, posicionamento de caixas de descarte, melhoria da iluminação dos ambientes, disponibilização de suportes reguláveis, implementação de sistema de segurança contra incêndios, manutenção de aparelhos básicos dentre outros.

O estudo de Pedrosa parte do pressuposto de que a infraestrutura das unidades básicas de saúde interfere na assistência prestada pelo enfermeiro. E apontam que as carências existentes podem comprometer o trabalho das equipes e os resultados operacionais. Esta autora considera a estrutura como um instrumento que pode indicar tendências gerais de qualidade, e que uma boa estrutura, com suficientes recursos e um desenho adequado do sistema, é, provavelmente, uma forma de garantir e promover a qualidade da atenção ${ }^{15}$.

Assim, investir na infraestrutura das unidades além de proporcionar segurança e conforto aos profissionais, significa a melhoria da qualidade da assistência prestada. E também a valorização do profissional, que estará munido dos recursos necessários para uma boa prática. Não sendo necessário desgaste profissional no uso de improvisos e adequações.

\section{Equipamento de Proteção Individual}

Os Equipamentos de Proteção Individual (EPI) são de suma importância para diminuição da exposição aos riscos, principalmente em relação aos riscos biológicos. Além do uso dos EPIs, os artigos enfatizaram a necessidade das instituições de saúde disponibilizarem esses equipamentos em quantidade suficiente, tamanhos adequados e de qualidade elevada. Essas recomendações devem ser aderidas e a atenção às necessidades dos funcionários deve ser priorizada6.

A Norma Regulamentadora de número 6 dispõe sobre o uso de Equipamentos de Proteção Individual afirma que a empresa é obrigada a fornecer aos empregados gratuitamente o EPI adequado ao risco, em perfeito estado de conservação e funcionamento ${ }^{16}$.

A mesma norma também determina que é de responsabilidade da instituição orientar e treinar o trabalhador sobre o uso adequado dos EPIs, a maneira adequada de guardar e conservação do material a fim de que o mesmo não perca a função original ${ }^{16}$. É necessário destacar que as precauções padrão podem ajudar a reduzir, mas não eliminam o risco de exposição ocupacional?.

\section{Participação dos trabalhadores}

Os trabalhadores são os sujeitos que mais conhecem os desafios de sua prática. Os artigos trazem a participação 
dos trabalhadores como estratégia para levantar os principais pontos a serem desenvolvidos nas instituições. A coparticipação dos trabalhadores nas decisões relativas aos riscos ocupacionais valorizam o profissional, que se sente ouvido pela instituição e participante no processo de implementação de melhorias ${ }^{17}$.

Na perspectiva de compreender o processo de trabalho real e apontar estratégias que previnam determinados agravos em saúde, os gestores deverão estar atentos ao processo de trabalho, incluindo a participação dos trabalhadores no debate e implementação de normas em saúde, pois não basta a racionalidade técnica, é preciso considerar as racionalidades da atividade real do trabalhador ${ }^{17}$.

Os saberes expressos pelos sujeitos que realizam o trabalho, com foco na exposição aos riscos ocupacionais podem ser relevantes à medida que se faz um diagnóstico mais completo do processo-saúde-doença no trabalho. $\mathrm{E}$ se investiga o problema por óticas diferentes. Não somente sob o olhar do pesquisador, mas também sob o olhar de quem vivencia a problemática ${ }^{17}$.

\section{Realização de campanhas de Imunização}

A imunização foi apontada por quatro produções (14\%) como principal forma de prevenção aos riscos biológicos. A realização de campanhas de vacinação, e a triagem sorológica para Hepatite $B$ foram sugeridas pelas publicações ${ }^{12-18}$.

A vacina para hepatite $B$ é uma das principais medidas de prevenção pré-exposição, sendo extremamente eficaz com 90 a $95 \%$ de resposta vacinal em adultos imunocompetentes, oferecida gratuitamente na rede pública de saúde desde o ano de $1995^{18}$.

Apesar disso ainda encontram-se profissionais que ignoram a vacinação, ou que possuem esquema vacinal incompleto, colocando em risco a sua saúde. O artigo 168 da Lei 7.855/89 obriga a realização de exames admissionais com a comprovação de esquema vacinal completo contra $\mathrm{HBV}^{19}$. Portanto, esses dados demonstram o não cumprimento da legislação no que refere aos exames admissionais pelos contratantes.

\section{Melhoria das Condições de Trabalho dos Profissionais}

A categoria Melhoria das Condições de Trabalho dos Profissionais, diferentemente da categoria Melhoria Estrutural, está voltada exclusivamente para os profissionais, considerando fatores como a jornada de trabalho e remuneração.

As publicações apontam a necessidade de adequação de carga horária, compensação justa além de boas condições de segurança e saúde laboral. A captação de recursos materiais e humanos também é apresentada como maneira eficiente para melhorar as condições de trabalho através da redução da sobrecarga de trabalho. Essa captação deve atender as necessidades reais da organização e dos trabalhadores ${ }^{5-6}$.

O profissional de enfermagem é exposto a condições de trabalho por vezes sacrificantes. O excesso de empregos é justificado pela baixa remuneração. Nestas circunstâncias, o trabalho torna-se transtorno psicológico para o profissional $^{5-6}$.

As dificuldades para os trabalhadores de enfermagem suportarem as cargas pesadas de trabalho, configuramse em alto risco para erros, visto que a sobrecarga produz cansaço físico e mental, reduzindo a atenção e comprometendo, portanto, a segurança dos próprios profissionais ${ }^{5-6}$

\section{Estímulo a Notificação de Acidentes de trabalho}

A cultura de notificação deve ser difundida a fim de divulgar aos órgãos responsáveis a realidade do trabalho da enfermagem. A notificação dos acidentes de trabalho revela o perfil e a ocorrência dos acidentes no local de trabalho, apontando para a necessidade de intervenções eficazes.

Enquanto os profissionais de saúde não se conscientizarem da importância da notificação do acidente, a dimensão real do problema não poderá ser determinada ${ }^{7}$. Os autores também afirmam que:

quando se consegue coletar todas as informações a respeito de um acidente, podem-se analisar os dados para priorizar esforços e estabelecer metas em unidades de maior risco, identificando as causas, no sentido de determinar se as ocorrências requerem aperfeiçoamento técnico na realização dos procedimentos, na aquisição de dispositivos mais seguros, na mudança de comportamento de funcionários ou se há falhas no suprimento de equipamentos de proteção individual e coletiva $a^{7: 275}$.

\section{CONCLUSÃO}

O estudo identificou alta frequência de publicações voltadas para riscos biológicos, e semelhantemente, o número de publicações desenvolvidas em unidades hospitalares também foi expressivo. Acredita-se que haja correlação entre os mesmos, à medida que o risco biológico é a principal preocupação em unidades de atenção terciária e quaternária pela qualidade e quantidade de procedimentos realizados.

A recomendação mais expressiva como estratégia para redução ou eliminação da exposição aos riscos ocupacionais pela equipe de enfermagem foi a intervenção educativa, com mais de $53 \%$ de intervalo entre a segunda recomendação mais frequente. Este resultado é claro para a credibilidade dada a ações educativas e destacam o poder transformador da mesma ao empoderar profissionais para os desafios práticos do cotidiano ocupacional.

$\mathrm{O}$ estudo respondeu aos seus objetivos e apresentou as recomendações sobre a exposição aos riscos ocupacionais 
para os profissionais de enfermagem sugeridas pela literatura. Os determinantes que podem levar o trabalhador da saúde ao adoecimento ou acidente de trabalho por exposição aos riscos ocupacionais, correspondem por um conjunto de condições, que podem ser reduzidas com a aplicação de intervenções eficazes. Muitas recomendações são apresentadas, e cabe às instituições colocarem em prática as que melhor atendam suas necessidades.

A escolha da melhor intervenção a ser aplicada deve considerar as características estruturais, humanas e os recursos disponíveis para sua implementação. Esta pesquisa estimula outras produções a investirem seus esforços em testarem a eficácia das recomendações aqui apresentadas, a fim de corroborar com os artigos que as propuseram, ou de apresentar novas intervenções que reduzam a exposição dos profissionais de enfermagem aos riscos ocupacionais.

Desta forma, as pesquisas sobre risco ocupacional com a equipe de enfermagem preencherão as lacunas de conhecimento, galgando novos rumos, construindo novos conhecimentos e novas formas de pesquisar em enfermagem.

\section{REFERÊNCIAS}

1. Organização Mundial de Saúde (OMS). Constituição da Organização Mundial da Saúde. Nova lorque (NY): Biblioteca Virtual de Direito da USP; 1946.

2. Ministério da Previdência Social (BR). Anuário Estatístico da Previdência Social [Internet]. 2013 [acesso em 24 abr 2015]; 4(1):1-12. Disponível em: http://www.previdencia.gov.br/ wp-content/uploads/2015/03/AEPS-2013-v-26.02.pdf.

3. Ministério da Saúde (BR). Doenças relacionadas ao trabalho: manual de procedimentos para os serviços de saúde [Internet]. 2001 [acesso em 23 abr 2015]; 114(1):23-78. Disponível em: http://bvsms.saude.gov.br/bvs/publicacoes/ doencas_relacionadas_trabalho1.pdf.

4. Botelho LLR, Cunha CCA, Macedo M. O Método da Revisão Integrativa nos Estudos Organizacionais. Gestão e Sociedade [Internet]. 2011 [acesso em 23 abr 2015]; 5(11): 121136. Disponível em: http://www.gestaoesociedade.org/ gestaoesociedade/article/viewFile/1220/906.

5. Beleza CMF, Gouveia MTO, Robazzi MLCC, Torres CRD, Azevedo GAV. Riscos Ocupacionais e Problemas de Saúde Percebidos por Trabalhadores de Enfermagem em Unidade Hospitalar. Cienc. enferm. [Internet]. 2013 [acesso em 27 out 2014]; 19(3):63-71. Disponível em: http://www. scielo.cl/scielo.php?script=sci_arttext \&pid=S0717$95532013000300008 \& \operatorname{lng}=e s \& n r m=i s o$.

6. Souza NVDO, Pires AS, Gonçalves FGA, Cunha LS, Shoji $\mathrm{S}$, Ribeiro LV, et al. Riscos ocupacionais relacionados ao trabalho de enfermagem em uma unidade ambulatorial especializada. Rev. enferm. UERJ [Internet]. 2012 [acesso 28 out 2014]; 20(esp.1):609-14. Disponível em: http://www. facenf.uerj.br/v20nesp1/v20e1a10.pdf

7. Machado MRM, Machado FA. Acidentes com material biológico em trabalhadores de enfermagem do Hospital Geral de Palmas (TO). Rev. bras. saúde ocup. [Internet]. 2011 [acesso em 22 out 2014]; 36(124)274-281. Disponível em: http:// www.scielo.br/scielo.php?script=sci_arttext \& pid=S0303$76572011000200011 \&$ lng=en \&nrm=iso.

8. Lima LM, Oliveira CC, Rodrigues KMR. Exposição ocupacional por material biológico no Hospital Santa Casa de Pelotas-2004 a 2008. Esc. Anna Nery. [Internet]. 2011 [acesso em 28 out 2014]; 15(1):96-102. Disponível em: http:// www.scielo.br/scielo.php?script=sci_arttext \&pid=S141481452011000100014 \&lng=en \&nrm $=$ iso.

9. Morais EN, Soares E, Lamas AR. Ferramenta para o gerenciamento preventivo dos riscos ocupacionais dos trabalhadores de enfermagem: mapa de riscos. R. pesq.: cuid. fundam. Online [Internet]. 2010 [acesso em 1 nov 2014] 2(3):1039-1047. Disponível em: http://www.seer.unirio.br/ index.php/cuidadofundamental/article/view/596/pdf_41.

10. Santos JLG, Vieira M,Assuiti LFC, Gomes D, Meirelles BHS, Silva MAS. Risco e vulnerabilidade nas práticas dos profissionais de saúde. Rev. Gaúcha Enferm. [Internet]. 2012 [acesso em 26 jun 2015]; 33(2): 205-212. Disponível em: http:// www.scielo.br/scielo.php?script=sci_arttext \& pid=S1983$14472012000200028 \&$ lng $=$ en.

11. Santos AS, Araújo TME, Viana MRP, Santos MS, Araújo RRM, Campelo TPT. Acidentes perfurocortantes em profissionais de enfermagem de serviços de urgência e emergência em uma capital brasileira. R. pesq.: cuid. fundam. online [Internet]. 2011 [acesso 28 out 2014]; (Ed.Supl.):229241. Disponível em: http://www.seer.unirio.br/index.php/ cuidadofundamental/article/view/1986/pdf_539.

12. Claudio CV, Sarquis LMM, Scussiato LA, Miranda FMA. Monitoramento biológico sob a ótica dos enfermeiros gerentes. Rev Rene. [Internet]. 2013 [acesso em 27 out 2014]; 14(2):252-61. Disponível em: http://www.revistarene.ufc.br/ revista/index.php/revista/article/view/180/pdf.

13. Simão SAF, Soares CRG, Souza V, Borges RAA, Cortez EA. Acidentes de trabalho com material perfurocortante envolvendo profissionais de enfermagem de unidade de emergência hospitalar. Rev. enferm. UERJ [Internet]. 2010 [acesso em 29 out 2014]; 18(3):400-4. Disponível em: http:// www.facenf.uerj.br/v18n3/v18n3a11.pdf.

14. Sardinha Peixoto L, Gonçalves LC, Costa TD, Tavares CMM, Cavalcanti ACD, Cortez EA. Educación permanente, continuada y de servicio: desvelando sus conceptos. Enferm. glob. [Internet]. 2013 [acesso em 10 jun 2015]; 12(29). Disponível em: http:// scielo.isciii.es/scielo.php?script=sci_arttext \&pid=S169561412013000100017\&lng=es\&nrm=iso.

15. Pedrosa ICF, Corrêa ACP, Mandú ENT. Influências da Infraestrutura de Centros de Saúde nas Práticas Profissionais: Percepções de Enfermeiros. Cienc.Cuid.Saude. [Internet]. 2011 [acesso em 12 jun 2015]; 10(1):058-065. Disponível em: http://periodicos.uem.br/ojs/index.php/CiencCuidSaude/ article/viewFile/13288/pdf.

16. Brasil. Ministério do Trabalho e Emprego. NR 6. Equipamento de Proteção Individual - EPI. Brasília: Ministério do Trabalho e Emprego [Internet]. 1978 [acesso em 14 mai 2015]. Disponível em: http://www.mte.gov.br/images/Documentos/ SST/NR/NR6.pdf.

17. Fontana RT. Situação de trabalho da enfermagem em uma instituição de atenção básica à saúde: saberes e práticas. [Tese de doutorado]. Porto Alegre (RS): Universidade Federal 
do Rio Grande do Sul [Internet]. 2011 [acesso em 2 nov 2014]. Disponível em: http://www.lume.ufrgs.br/bitstream/ handle/10183/38860/000824108.pdf?sequence=1.

18. Fraguás SA, Silvino ZR, Flach DMAM, Couto IRR, Andrade M. Imunização Contra Hepatite B: Uma questão de Saúde do Trabalhador de Enfermagem. R. pesq.: cuid. fundam. [Internet]. 2013 [acesso em 21 out 2014]; 5(1):3150-58. Disponível em: http://www.seer.unirio.br/index.php/cuidadofundamental/ article/view/1865/pdf_671.

19. Brasil. Lei $n^{\circ} 7.855$, de 24 de Outubro de 1989. Altera a Consolidação das Leis do Trabalho, atualiza os valores das multas trabalhistas, amplia sua aplicação, institui o Programa de Desenvolvimento do Sistema Federal de Inspeção do Trabalho e dá outras providências [Internet]. 1989 [acesso em 25 out 2014]. Disponível em: http://www3.dataprev.gov. br/sislex/paginas/42/1989/7855.htm.

20. Szeto GPY, Law KY, Lee E, Lau T, Chan SY, Law SW. Multifaceted ergonomic intervention programme for community nurses: pilot study. Journal of Advanced Nursing. [Internet]. 2010 [acesso em 23 out 2014] Disponível em:http://www.readcube. com/articles/10.1111\%2Fj.1365-2648.2009.05255.x?r3 referer=wol\&tracking_action=preview_click\&show_ checkout=1\&purchase_referrer=onlinelibrary.wiley. com \&purchase_site_license=LICENSE_DENIED_NO_ CUSTOMER.

21. Trindade JPA, Rodrigues EG, Sousa, TK, Palos MAP, Santos SLV. Trabajadores del área de salud de un hospital oncológico colonizados por microorganismos multidroga resistentes. Enfermería Global [Internet]. 2014 [acesso em 24 out 2014]; (33)227-239. Disponible en: http://scielo.isciii.es/pdf/eg/ v13n33/administracion2.pdf.

22. Silva EECM, Valença CN, Lima GAF, Oliveira MD, Germano RM, Rosanelli CLSP. Avaliação ergonômica do posto de urgência em uma unidade mista de saúde na cidade de Natal/Rio Grande do Norte.J. res.: fundam. care [Internet]. 2013 [acesso em 27 out 2014]; 5(3):227-34. Disponível em: http://www. seer.unirio.br/index.php/cuidadofundamental/article/ view/1999/pdf_857.

23. Baroni FCAL, Oliveria JCM, Guimarães GL, Matos SS, Carvalho DV. O trabalhador de enfermagem frente o gerenciamento de resíduo químico em unidade de quimioterapia antineoplásica. Rev Min Enferm [Internet]. 2013 [acesso em 23 out 2014]; 17(3): 560-564. Disponível em: http://www.reme.org.br/ artigo/detalhes/672.

24. Santos SS, Costa NA, Mascarenhas MDM. Caracterização das exposições ocupacionais a material biológico entre trabalhadores de hospitais no Município de Teresina, Estado do Piauí, Brasil, 2007 a 2011. Epidemiol. Serv. Saúde [Internet]. 2013 [acesso em 25 out 2014]; 22(1):165-170. Disponível em: http:// scielo.iec.pa.gov.br/scielo.php?script=sci_arttext \&pid=S167949742013000100017\&lng=pt\&nrm=iso.

25. Cavalcante CAA, Cavalcante EFO, Macêdo MLAF, Cavalcante ES, Medeiros SM. Acidentes com Material Biológico em Trabalhadores. Rev Rene. [Internet]. 2013 [acesso em 25 out 2014]; 14(5):971-9. Disponível em: http://www.revistarene. ufc.br/revista/index.php/revista/article/view/1267.

26. Valle ARMC, Moura MEB, Nunes BMVT, Figueiredo MLF. A Biossegurança sob o olhar de enfermeiros. Rev. enferm. UERJ [Internet]. 2012 [acesso em 24 out 2014]; 20(3):361-7.
Disponível em: http://www.e-publicacoes.uerj.br/index.php/ enfermagemuerj/article/view/4108.

27. Ruas EFG, Santos LS, Barbosa DA, Belasco AGS, Bettencourt ARC. Acidentes ocupacionais com materiais perfurocortantes em hospitais de Montes Claros-MG. Rev Min Enferm [Internet]. 2012 [acesso em 26 out 2014]; 16(3): 437-443. Disponível em: http://reme.org.br/artigo/detalhes/547.

28. Ghodsbin F, Bijani M, Rahmati H, Mohebbi Z, Kamali M. Effect of education on the incidence rate of occupational exposure resulting from sharp bodies and mucocutaneous contamination with blood and body fluids of patients among nursing personnel of Valiasr Hospital- Fassa, 2008. Invest Educ Enferm. [Internet]. 2011 [acesso em 29 out 2014]; 29(1):61-67. Disponível em: http://aprendeenlinea.udea. edu.co/revistas/index.php/iee/article/view/8525/7850.

29. Nunes MBG, Robazzi MLCC, Terra FS, Mauro MYC,Zeitoune RCG, Secco IAO. Riscos ocupacionais dos enfermeiros atuantes na atenção à saúde da família. Esc. Anna Nery [Internet]. 2010 [acesso em 22 out 2014]; 18(2): 204-9. Disponível em: http:// www.facenf.uerj.br/v18n2/v18n2a07.pdf.

30. Luckwü ADGV, Silva EL, Araújo EC. Fatores de exposição do profissional da saúde com substâncias químicas utilizadas nos processos de lavagem e desinfecção no expurgo. Rev enferm UFPE on line [Internet]. 2010 [acesso em 28 out 2014]; 4(1):254-61. Disponível em: http://www.revista. ufpe.br/revistaenfermagem/index.php/revista/article/ viewFile/750/pdf_318.

31. Silva LF, Reis PED. Avaliação do conhecimento da equipe de enfermagem sobre riscos ocupacionais na administração de quimioterápicos. Revista Brasileira de Cancerologia [Internet]. 2010 [acesso em 1 nov 2014]; 56(3):311-320. Disponível em: http://www1.inca.gov.br/rbc/n_56/v03/pdf/04_artigo_ avaliacao_conhecimento_equipe_enfermagem_riscos_ ocupacionais_administracao_quimioterapicos.pdf.

32. Simao SAF, Souza V, Borges RAA, Soares CRG, Cortez EA. Fatores associados aos acidentes biológicos entre profissionais de enfermagem. Cogitare enferm. [Internet]. 2010 [acesso em 1 nov 2014]: 15(1): 87-91. Disponível em: http://www. revenf.bvs.br/scielo.php?script=sci_arttext \& pid=S1414$85362010000100013 \&$ lng=es \& nrm=iso.

33. Duarte NL, Mauro MYC. Análise dos fatores de riscos ocupacionais do trabalho de enfermagem sob a ótica dos enfermeiros. Rev. bras. saúde ocup. [Internet]. 2010 [acesso em 2 nov 2014]; 35(121):157-167. Disponível em: http:// www.scielo.br/scielo.php?script=sci_arttext \&pid=S030376572010000100017 \&lng=en \&nrm=iso.

34. Cunha AC, Mauro MYC. Educação Continuada e a Norma Regulamentadora 32:utopia ou realidade na enfermagem?.Rev. bras. saúde ocup. [Internet]. 2010 [acesso em 2 nov 2014]; 35(122):305-313. Disponível em: http://www.scielo.br/scielo. php?script=sci_arttext \&pid=S0303-76572010000200013.

35. Dalri RCMB, Robazzi MLCC, Silva LA. Riscos ocupacionais e alterações de saúde entre trabalhadores de enfermagem brasileiros de unidades de urgência e emergência. Cienc. enferm. [Internet]. 2010 [acesso em 2 nov 2014]; 16(2):69-81. Disponível em: http://www.scielo.cl/pdf/cienf/v16n2/art_08.pdf.

36. Gallas SR, Fontana RT. Biossegurança e a enfermagem nos cuidadosclínicos:contribuições paraa saúde do trabalhador.Rev. bras. enferm. [Internet]. 2010 [acesso em 3 nov 2014]; 
63(5):786-792. Disponível em: http://www.scielo.br/scielo. php?script=sci_arttext\&pid=S0034-71672010000500015.

37. Polovich M, Clark PC. Nurses' use of hazardous drug safe handling precautions. [Dissertação]. Georgia (USA): Georgia State University [Internet]. 2010. [acesso 4 nov 2014]. Disponível em: http://scholarworks.gsu.edu/cgi/ viewcontent.cgi?article $=1010 \&$ context $=$ nursing_diss 